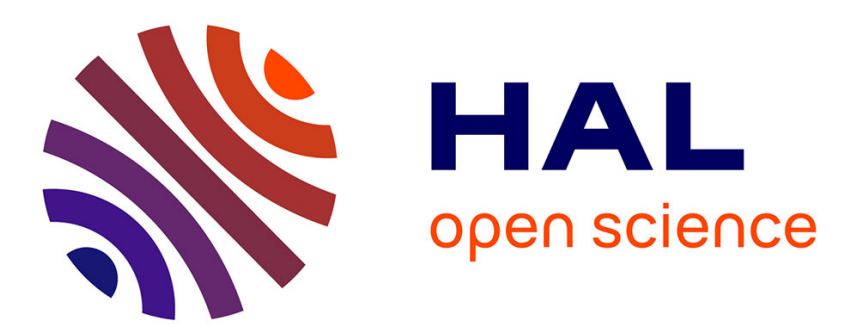

\title{
The influence of audiovisual stimuli cuing temperature, carbonation, and color on the categorization of freshness in beverages
}

Jérémy Roque, Jérémie Lafraire, Charles Spence, Malika Auvray

\section{- To cite this version:}

Jérémy Roque, Jérémie Lafraire, Charles Spence, Malika Auvray. The influence of audiovisual stimuli cuing temperature, carbonation, and color on the categorization of freshness in beverages. Journal of Sensory Studies, 2018, 33 (6), pp.e12469. 10.1111/joss.12469 . hal-02393381

\section{HAL Id: hal-02393381 \\ https://hal.science/hal-02393381}

Submitted on 4 Dec 2019

HAL is a multi-disciplinary open access archive for the deposit and dissemination of scientific research documents, whether they are published or not. The documents may come from teaching and research institutions in France or abroad, or from public or private research centers.
L'archive ouverte pluridisciplinaire HAL, est destinée au dépôt et à la diffusion de documents scientifiques de niveau recherche, publiés ou non, émanant des établissements d'enseignement et de recherche français ou étrangers, des laboratoires publics ou privés. 


\title{
The influence of audiovisual stimuli cuing temperature, carbonation, and color on the categorization of freshness in beverages
}

\author{
Jérémy Roque ${ }^{1,2,3}$ ๑ | Jérémie Lafraire ${ }^{2} \mid$ Charles Spence $^{4}$ (1) | Malika Auvray ${ }^{3}$
}

\author{
${ }^{1}$ Breakthrough Innovation Group, Pernod \\ Ricard, Paris, France \\ ${ }^{2}$ Institut Paul Bocuse Research Center, Ecully, \\ France \\ ${ }^{3}$ Sorbonne Université, UPMC, CNRS, Institut \\ des Systèmes Intelligents et de Robotique \\ (ISIR), Paris, France \\ ${ }^{4}$ Crossmodal Research Laboratory, \\ Department of Experimental Psychology, \\ Oxford University, Oxford, United Kingdom \\ Correspondence \\ Jérémy Roque, Centre de Recherche Institut \\ Paul Bocuse, 1 Chemin de Calabert, 69130 \\ Ecully, France. \\ Email: jeremy.roque@pernod-ricard.com \\ Funding information \\ Association Nationale de la Recherche et de la \\ Technologie, Grant/Award Number: \\ 2015/0894; Pernod Ricard
}

\begin{abstract}
The study reported here investigated the influence of audiovisual stimuli signaling the likely temperature (the presence versus absence of ice cubes), the likely level of carbonation (the presence versus absence of bubbles), and the color of the liquid on the categorization of freshness in beverages. Participants made speeded categorization responses ("fresh" versus "not fresh") concerning the bimodal stimuli. When the stimuli were categorized as fresh, visible ice cubes decreased the participants' reaction times (RTs) the most, followed by the sound of ice cubes, and then the sound of carbonation. Overall, the participants categorized more the stimuli as fresh in the presence of ice cubes visible in the drink. When presented together, the targeted audiovisual perceptual features exerted an additive effect in both decreasing RTs and increasing the likelihood that beverages would be categorized of fresh. No significant effect of beverage color (manipulated betweenparticipants) was observed. These results are discussed in terms of the crossmodal interaction effects that might be expected to influence the multisensory experience of freshness in beverages.

\section{Practical applications}

The current appeal of consumers for a global experience of freshness in the food and beverage domain constitutes a promising lever for strategic innovation. Thus, it appears timely to investigate how some sensory cues other than the chemosensory ones (i.e., taste and smell) involved in a multisensory drink experience, namely visual and auditory cues, interact and subsequently impact consumers' perception and behavior. We believe that the triggering of specific cognitive mechanisms, which may occur during the multisensory integration processes associated with freshness perception could help to increase beverages' attractiveness and appreciation. The present study thus has important implications for product formulation and marketing design purposes.
\end{abstract}

\section{1 | INTRODUCTION}

\subsection{Multisensory contributions to the perception of freshness}

Over the past decade, the perception of freshness has received consideration in consumer studies since it is generally perceived as pleasurable by consumers and it thus may be expected to influence their purchase and consumption behavior (see Eccles, Du-Plessis, Dommels, \& Wilkinson, 2013; Labbe et al., 2009; Roque, Auvray, \& Lafraire, 2018, for reviews). Considering the particular case of beverages, it should be noted that the concept of freshness may convey different meanings because it can refer to: (a) the overall multisensory experience during a drinking episode (involving for instance coldness, sourness, or a menthol odor that will contribute to an actual perception of freshness, but also some visual cues for instance, that may trigger freshness expectations), (b) the aging of the organic ingredients contained in the drink (e.g., aging of the mint leaves in a mojito), or else (c) the time delay (informed and/or perceived) to which the drink has been prepared before being served. Hence, it is challenging to disentangle behaviorally the distinct, though likely related, meanings of freshness since the three meanings reported above may well overlap. Based on previous research (see Labbe, Almiron-Roig, et al., 2009; Roque et al., 2018 for reviews), it appears that the multisensory experience of freshness is likely built from two fundamental cognitive mechanisms that include: (a) expectations elicited by visual, auditory, and orthonasal olfactory cues for which a product can be perceived as 
fresh, before tasting, and (b) actual sensations elicited by gustatory and olfactory cues, as well as peripheral nervous systems including oral-somatosensory, nociceptive, and tactile cues contributing to the perception of freshness itself during tasting. As a first investigation of the crossmodal interaction effects that likely influence the categorization of particular beverages as being fresh, we chose to focus in this study on visual and auditory cues that may generate expectations of freshness.

The majority of previous studies that have investigated the multisensory perception of freshness have focused on the influence of gustatory, olfactory, and oral-somatosensory cues.

Some of this research highlights different patterns of sensory dominance regarding the various sensory inputs contributing to freshness. For instance, Fenko, Schifferstein, Huang, and Hekkert (2009) documented that for soft drinks and dishwashing liquids, olfactory cues dominated over visual color cues as far as freshness judgments were concerned. Labbe, Gilbert, Antille, and Martin (2009), meanwhile, used edible gels, varying in their olfactory (mint and peach), trigeminal (coldness), taste (acidity), and texture (thickness) properties. The results of the latter study highlighted the fact that people differed in the main sensory modality that they associated with freshness, and that this appeared to be based on previously learned associations. One cluster of consumers assessed the contribution of smell (i.e., mint) and trigeminal (i.e., coldness) sensations to freshness as being most important. A second cluster of consumers considered taste (i.e., acidity) most important, whereas a third cluster ranked oralsomatosensory (i.e., low thickness for liquids) as the most important.

In another study, Westerink and Kozlov (2004) investigated what constitutes freshness for oral-care products by means of structured interviews with native English speakers. The results allowed for the identification of six main attributes as part of the concept of freshness: cleanness (related to mouth-cleaning activities), energy (related to energizing sensations experienced by the participants, for example, texture or bubbles), "water-ness" (related to the multiple experiences of having water in the mouth, that is, "after drinking water" feelings, or the natural level of salivation), coldness (related to temperature), taste, and smell. From these results, Westerink and Kozlov went on to argue that freshness is a dynamic concept that varies over time and individuals likely attribute different weights to the six attributes during product usage. Their results also highlight how people tend to value temporary perceptual features during the actual experience of freshness (e.g., temperature and energy), whereas they tend to keep other sensory features related to the perception of freshness (e.g., particular compounds such as menthol) in long-term memory.

The complexity of freshness highlighted by these previous studies represents an additional argument to investigate first the influence of audiovisual perceptual features and their crossmodal interaction effects that may trigger freshness expectations before the actual consumption experience. When specifically focusing on the multisensory perception of freshness in beverages, there is a research gap with regard to the way audiovisual crossmodal interaction effects may facilitate the categorization of a particular beverage as being fresh or not. Some audiovisual features have nonetheless been highlighted as potential modulators of the multisensory drinking experience and they therefore represent good candidates to investigate further.

\subsection{Audiovisual perceptual features involved in a multisensory drinking experience and their potential influence on freshness}

According to consumers, coldness is one of the common sensory properties that enhances freshness perception, through different sensory cues (i.e., oral-somatosensory, tactile, trigeminal, as well as visual and olfactory cues), in food and beverages (Eccles et al., 2013; Labbe, Almiron-Roig, et al., 2009; Zellner \& Durlach, 2002), water (Labbe, Martin, Le Coutre, \& Hudry, 2011), and soft drinks (McEwan \& Colwill, 1996; Saint-Eve et al., 2010; Zhang, Lusk, Mirosa, \& Oey, 2016). Guinard, Souchard, Picot, Rogeaux, and Sieffermann (1998) have also highlighted that the density of visual bubbles positively increases the perceived freshness in beers even though the foam created by bubbles on the top of the liquid can exert a negative impact on such judgments.

Regarding the influence of auditory cues, recent studies have highlighted the influence of sounds corresponding to the pouring of a beverage on the perception of its temperature (Velasco, Jones, King, \& Spence, 2013). The results of the latter study revealed the existence of a crossmodal correspondence (see Section 1.3) between the pouring sounds and a particular temperature: the sonic properties of the sounds of hot and cold waters were, respectively, associated with congruent words attributes (i.e., "Hot Drink" and "Cold Drink"; see also Wang \& Spence, 2017). Another study conducted by Zampini and Spence (2005) highlighted that the perception of carbonation in a beverage may be influenced by the auditory cues provided by the bubbles (note that drinks packaging opening sounds may also be relevant here; see Spence, 2015; Spence \& Wang, 2017). Hence, the sound of carbonation is likely to influence people's perception of freshness in beverages as well.

Finally, it is worth mentioning here the influence of color on the perception of freshness in beverages. To date, there is a lack of consensus in the literature concerning whether a particular color or group of colors are mainly associated to fresh beverages by consumers. Some studies have nonetheless reported a noticeable exception for clear beverages which are favored, in contrast to dark ones, when consumers have to characterize the appropriate color for fresh beverages (Clydesdale, Gover, Philipsen, \& Fugardi, 1992; Zellner \& Durlach, 2002, 2003). However, none of these studies detailed what consumers meant by "clear color" (for instance in terms of transparency or saturation). Additional early results obtained by Zellner and Durlach (2003) revealed that freshness ratings attributed to different colors of a vanilla-flavored beverage significantly differed for expected freshness ratings, whereas freshness ratings did not differ significantly from one another when the participants actually tasted the beverages. Meanwhile, another study conducted by Clydesdale et al. (1992) revealed that American students most frequently associated clear (36\%), followed by brown (24\%), red (17\%), and orange (12\%) colors with the thirst-quenching character of fruit punchflavored beverages. Importantly for present purposes, the thirstquenching properties of beverages have been shown to correlate positively with the perception of freshness (e.g., Labbe, Gilbert, et al., 2009) and thereby they constitute a meaningful indicator of freshness. To summarize, it would appear that various factors can modulate the influence of color on perceived freshness. For instance, the color of a beverage can set freshness expectations that will not necessarily 
impact freshness ratings during tasting (e.g., Carvalho, Moors, Wagemans, \& Spence, 2017). This discrepancy may be attributable to other sensory contributions (e.g., smell, taste, or flavor) and it likely depends on the past learned associations of consumers.

The results of the studies described above stress the fact that different audiovisual perceptual features (e.g., perceptual cues linked to coldness, carbonation, and color) occurring prior to tasting influence freshness expectations and may thereafter, in some cases at least, be expected to influence the final perception of freshness. In terms of methodology, the majority of studies investigating the concept of freshness have focused on consumers' expectations by a collection of declarative data. Given the complexity of the cognitive mechanisms at hand in freshness perception, this approach needs to be completed by more objective measures of behavior such as categorization performance. A growing literature has highlighted the existence of specific cognitive mechanisms, namely crossmodal correspondences, which induce measureable crossmodal interaction effects. Thus, the next section is devoted to presenting why considering such mechanisms is relevant in the case of freshness in beverages.

\section{3 | Crossmodal correspondences and the measure of audiovisual crossmodal interaction effects}

Audiovisual crossmodal interactions have been widely studied in research dealing with multisensory integration. In particular, the evidence that has been published to date points to the existence of numerous crossmodal correspondences. Crossmodal correspondences have been defined as the nonarbitrary associations that exist between different perceptual features belonging to various sensory modalities (see Spence, 2011, for a review). In a number of cases, crossmodal correspondences have been shown to lead to perceptual consequences such as crossmodal enhancement at different stages of human information processing (e.g., Liang, Roy, Chen, \& Zhang, 2013). Moreover, it has been shown that a number of crossmodal correspondences are shared across individuals and appear to be consistent over time (Spence, 2011). The existence of specific crossmodal correspondences are often inferred from participants' reaction times (RTs) using speeded classification tasks, including variants of the Implicit Association Test to determine the strength of the association between two perceptual features (e.g., Demattè, Sanabria, \& Spence, 2007; Deroy, Fasiello, Hayward, \& Auvray, 2016; Parise \& Spence, 2012). This test is based on the rationale that when two stimuli share a strong association (i.e., congruent versus incongruent associations, see Roque et al., 2018), participants' RTs are shorter and their responses tend to be more accurate. More straightforward speeded classification tasks are also often used to investigate interaction effects between different perceptual features (e.g., Evans \& Treisman, 2011; Marks, 2004). A decrease in participants' RTs and a higher accuracy in their responses may reflect the triggering of participants' motor responses due to particular sensory inputs that belong to a category they are responding to (see Diederich \& Colonius, 2004). In the food and beverage domain, a large number of studies have been conducted to investigate the crossmodal interaction effects between various sensory modalities (e.g., see Knöferle \& Spence, 2012 for a review of crossmodal correspondences between musical sounds and basic tastes). However, there is still a lack of information on the interaction effects between visual and auditory cues that may occur before the actual consumption, but which still indirectly influence the subsequent consumers' experiences by triggering specific sensory expectations (see PiquerasFiszman \& Spence, 2015, for a review).

From an applied perspective, a better understanding of how to tap into the crossmodal correspondences regarding freshness would enable marketers, for instance, to facilitate consumers' categorization of a given product as fresh. This might well be expected to have a positive impact on consumers' product experience since freshness features are part of consumers' sensory expectations and thereby they are likely to determine food and beverage attractiveness and appreciation. However, the crossmodal correspondences that can occur during the multisensory integration that leads to the perception of freshness, in the particular context of beverages, still remain to be explored.

The study reported here did not aim at highlighting proper crossmodal correspondences but rather to investigate whether some audiovisual perceptual features could induce crossmodal interaction effects and influence the categorization of freshness in beverages. We believe that it represents a promising step along the path to identifying the different perceptual features that could induce crossmodal correspondences effects.

The literature described above led us to formulate the following three hypotheses:

Hypothesis 1. Both visual and auditory perceptual features linked to temperature (presence versus absence of ice cubes ${ }^{1}$ ), and carbonation (presence versus absence of bubbles ${ }^{2}$ ) would influence the categorization of freshness in beverages.

Hypothesis 2. Two main crossmodal interaction effects are expected: (a) shorter RTs in the freshness categorization task for congruent combinations of auditory and visual stimuli that display the perceptual features of interest (e.g., presenting both the sight and sound of ice cubes) than for incongruent ones, and (b) a tendency to categorize these congruent stimuli as fresh.

Hypothesis 3. Even though there is no consensus in the literature, a significant influence of the color of the liquid on the categorization of freshness is also expected since different colors can lead to different expectations regarding the corresponding flavor of the liquid (at least when assessed in a within-participants experimental design), as well as different product categories to which the participants may associate a particular freshness intensity.

To investigate these three hypotheses, a speeded categorization task was conducted with audiovisual stimuli cuing the likely temperature (presence versus absence of ice cubes), the likely level of carbonation (presence versus absence of bubbles), and seven different colors of the liquid. The participants had to categorize the different bimodal stimuli corresponding to different beverages as "fresh" versus "not fresh."

\footnotetext{
${ }^{1}$ Ice cubes were selected as the most relevant perceptual feature of interest for which participants could infer the temperature of the drink, and consequently the expected perceived freshness in mouth, among the range of eligible sensory cues (e.g., condensation on the glass).

${ }^{2}$ Carbonation in our study refers to both bubbles present within the liquid and the foam on its surface.
} 


\section{2 | MATERIALS AND METHODS}

\section{1 | Participants}

Eighty-four French participants (50\% female) took part in this study with a mean age of 29.8 ranging from 19 to 59 years old. They were recruited through the database of the Institut Paul Bocuse Research Center. All reported normal or corrected-to-normal vision and audition. None of the participants had any particular expertise in beverages in terms of leisure activities, education, or professional expertise. Each individual session lasted approximately $25 \mathrm{~min}$ and the participants received a $10 €$ voucher to complete the study. All of the participants provided written informed consent prior to taking part in the study. The experiment was approved by the local ethical committee.

\subsection{Apparatus and stimuli}

\subsection{1 | Visual stimuli}

Pictures of a $200 \mathrm{ml}$ liquid served in a standard glass were created based on a full factorial design varying temperature (no ice cubes versus four rectangular ones), carbonation (carbonated transparent lemonade $6.2 \mathrm{~g} / \mathrm{L} \mathrm{CO}_{2}$ versus still water), and colors (colorless, blue, green, yellow, orange, brown, and red, created using food-coloring, see Supporting Information). Pictures were taken in the same laboratory conditions in order to control for lighting, room temperature, liquid temperature (i.e., ambient), size of ice cubes, $\mathrm{CO}_{2}$ content, and color cues in the various different conditions (see Figure 1).

\subsection{2 | Auditory stimuli}

The sounds of $250 \mathrm{ml}$ of liquid being poured into a glass were recorded for $2 \mathrm{~s}$ across the factorial design giving rise to four different sounds: (a) still liquid without ice cubes, (b) carbonated liquid without ice cubes, (c) still liquid with four rectangular ice cubes, (d) carbonated liquid with four rectangular ice cubes. The liquids were the same as those used for the visual stimuli (i.e., still water and lemonade $6.2 \mathrm{~g} / \mathrm{L}$ $\mathrm{CO}_{2}$ ). The pitch of the sounds were normalized to $1,200 \mathrm{~Hz}$ in order to avoid different associations of the pitch with different liquid temperatures (see Velasco et al., 2013) and the background noise was reduced using Audacity 2.1.3 software. ${ }^{3}$

${ }^{3}$ Initially, an online experiment was conducted through an internet-based testing tool (Qualtrics) with 84 North American participants (50\% female, mean age 33.8) in order to investigate the potential influence of the targeted stimuli (i.e., audiovisual perceptual features cuing temperature, carbonation, and color) on the perception of freshness. In this pilot study, the participants had to rank the perceived freshness of each bimodal stimulus corresponding to a particular beverage, on a 9-point Likert scale ranging from "Not fresh at all" to "Very fresh," as rapidly as possible. Viewing ice cubes had a significant positive effect on the participants' freshness ratings whatever the interaction with carbonation, $p<.001(b=0.64, t(1)=9.1$, effect size: Pearson's correlation coefficient $r=0.99$ ). However, the sound of ice cubes had a significant negative effect on the freshness ratings, $p<.001(b=-0.31, t(3)=-4.4$, effect size: $r=0.93)$. Both the sound $(b=0.46, t(4)=5.3$, effect size: $r=0.94)$ and sight $(b=0.1, t$ (2) $=0.97$, effect size: $r=0.57$ ) of carbonation significantly increased the participants' freshness ratings, $p<.001$. Moreover, this effect was additive when the two were presented together. The color of the beverage had no effect on the freshness ratings $(F=0.76)$. To be noted that the effect sizes " $r$ " reported in this study have been computed as Pearson's correlation coefficients, as advised by Field, Miles, and Field (2012). " $r$ " was computed as $r=\sqrt{ }\left(t^{2} /\left(t^{2}+d f\right)\right)$, where

\subsection{Design and procedure}

The experiment was conducted in a controlled laboratory setting at Institut Paul Bocuse Research Center. The software E-prime was used to record participants' RTs. All the visual and auditory stimuli were crossed based on a full factorial design giving rise to 16 different bimodal stimuli, of which 4 were congruent and 12 incongruent. Some bimodal stimuli displayed two perceptual features (i.e., one visual and one auditory, for instance, both the sight and sound of ice cubes for congruent interactions), others displayed three (i.e., one visual and two auditory or two visual and one auditory, for instance the sight of ice cubes and carbonation as well as the sound of ice cubes), and others displayed four perceptual features (i.e., two visual and two auditory). All of the bimodal stimuli were presented to the participants 10 times in a random order, giving rise to a total of 160 trials. Hence, a repeated-measures design was adopted with the visual and auditory perceptual features signaling temperature and carbonation displayed as within-participants factors. Since the influence of color on flavor expectations in beverages has been widely reported in the literature (e.g., Carvalho et al., 2017), and also in order to avoid fatigue due to the duration of the experiment, the color variable in this study varied as a between-participants factor ( $N=12$ participants per color, gender balanced). The alpha level was set at .05 for all statistical analyses. Statistical analyses were performed using $R$ 3.4.1.

Before starting the experimental session, the participants were asked whether they were thirsty or not. In case they declared that they were thirsty, a $200 \mathrm{ml}$ glass of still water was served. The participants sat on a chair $70 \mathrm{~cm}$ from the computer screen and wore headphones (Sony MDR-ZX110) for which the volume was adjusted during the training phase until each participant stated they reached a sufficiently audible level. In each trial, the participants started by looking at a fixation cross in the center of the screen for $1,000 \mathrm{~ms}$. Next, the bimodal stimulus was displayed for 2,000 ms time-out, and the participants' task was to categorize as quickly as possible whether they perceived "the drink served in the glass as: Fresh or Not fresh" in terms of the expected freshness in mouth as if they would have to consume these drinks. To do so, they had to press one of two response keys ( $D$ or $J$ ) on the computer keyboard with their left and right index. Emphasis was placed on rapid responding (note that there were no correct responses in the task). The two response keys were counterbalanced across participants (see Figure 2).

\section{3 | RESULTS}

\section{1 | The influence of audiovisual interactions on participants' RTs}

Those trials in which the participants failed to make a response before the trial was terminated (less than $3.75 \%$ of trials overall) were not analyzed. Linear mixed-effects models were fitted to the data with

$t$ stands for the individual $t$ values for each level of variables stored in the model and $d f$ contain the degrees of freedom. $r$ may thus range from 0 (no effect) to 1 (a perfect effect). The effect is considered as large when $r$ is equal or higher than 0.5 . When $r=0.5$, the effect accounts for $25 \%$ of the variance. 


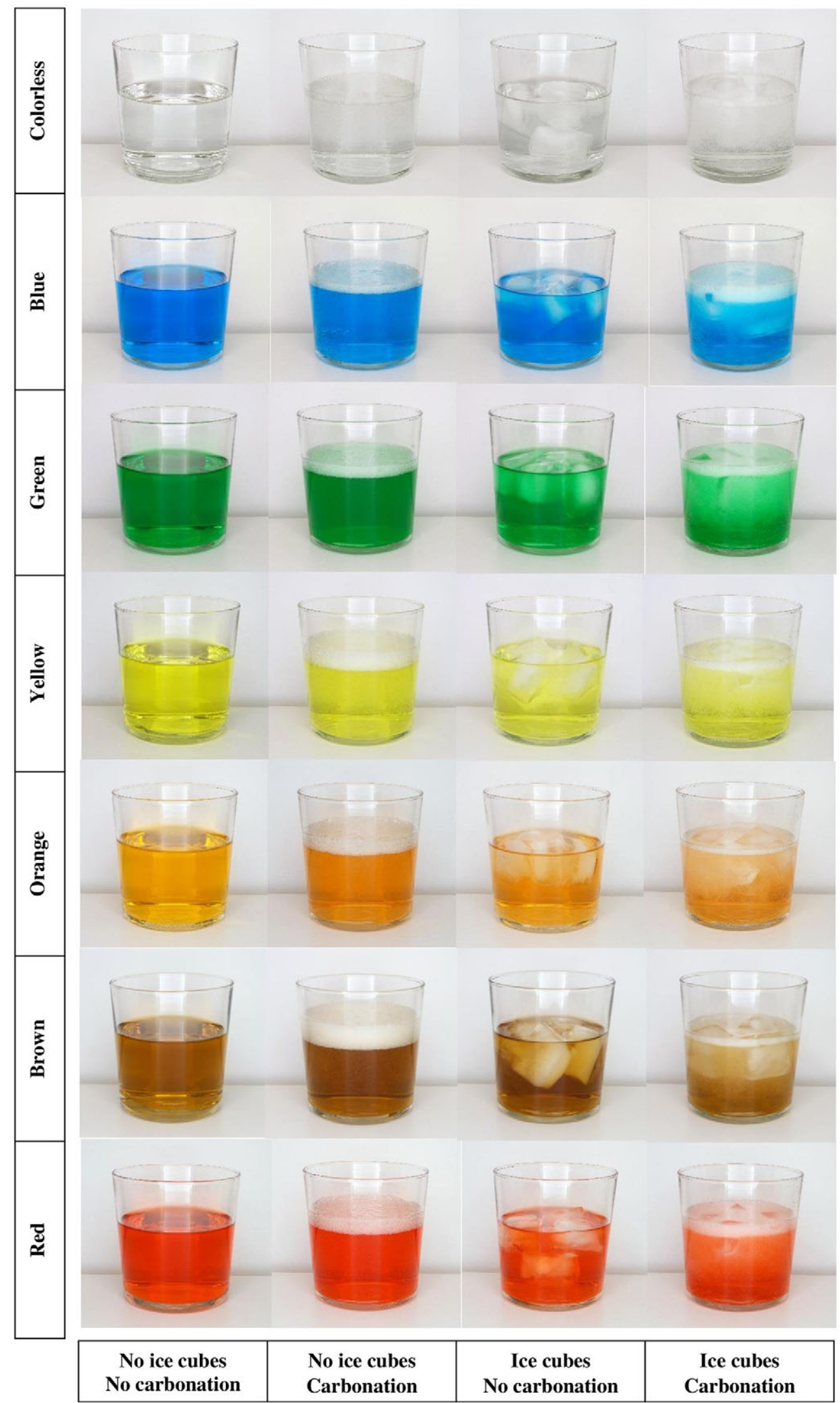

FIGURE 1 Pictures of the different visual stimuli used varying in temperature, carbonation, and color

participants as a random variable and the congruency variable as nested. A first model was conducted to test the overall effect of the congruency variable (i.e., congruent versus incongruent stimuli) on participants' RTs. This model $\left(R^{2}{ }_{C}=0.464\right.$, see Nakagawa \&
Schielzeth, 2013) revealed a significant main effect of congruency, $\chi 2$ $[1]=33.7, p<.0001$. Overall, the participants' RTs were significantly shorter for congruent stimuli ( $m=1,047 \mathrm{~ms} \pm 6.68 \mathrm{SEM}$ ) than for incongruent stimuli $(m=1,081 \mathrm{~ms} \pm 3.96 \mathrm{SEM})$. 


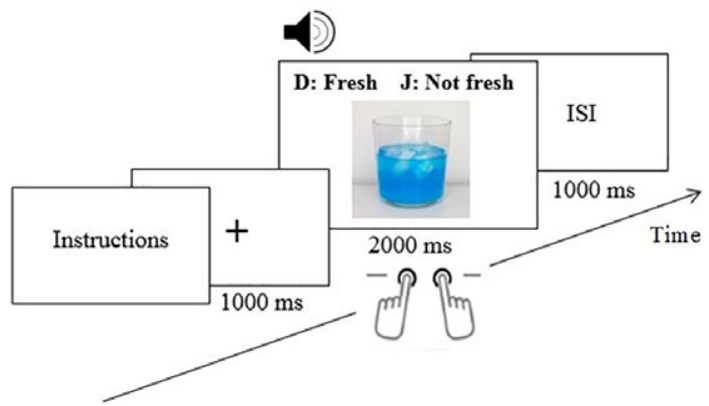

FIGURE 2 Timeline. ISI = intertrial stimulus interval. Note that the assignment of the two response keys was counterbalanced across participants

When considering the variables linked to the stimuli as independent variables and the participants' RTs as the outcome variable, the final model considered $\left(R^{2} \mathrm{C}=0.495\right)$ revealed a significant three-way interaction effect between the sight of ice cubes and the different carbonation conditions, $\chi 2(9)=18.51, p<.0001$. Another three-way interaction effect between the sound of ice cubes and the different carbonation conditions was revealed by the model, $\chi 2[12]=280.94$, $p<.0001$. The color of the stimuli did not significantly influence RTs $(\mathrm{F}=1.09, p=0.38)$. These results are presented in Figure 3.

The interaction between the sight of ice cubes and the different carbonation conditions appeared to have a significant influence on the participants' RTs: a Tukey post hoc analysis revealed that the sight of ice cubes in a drink always induced significantly faster RTs, as compared to the absence of ice cubes visually, whatever the interaction with carbonation, $p<.01(b=-74.11, t[5]=-6.13$, effect size: $r=0.94)$. When there was no carbonation, only a nonsignificant numerical tendency was observed ( $p=0.99$ ).

Another significant influence on the participants' RTs was revealed for the interaction between the sound of ice cubes and the different carbonation conditions: a Tukey post hoc analysis revealed that the sound of ice cubes only induced shorter RTs when interacting with the sound or both the sight and sound of carbonation, $p<.01$ ( $b=-149.7, t[7]=-12.46$, effect size: $r=0.98)$.

An additional result can be highlighted from these two graphs: the sight of carbonation always induced significantly longer RTs, $p<.01(b=96.42, t[6]=7.41$, effect size: $r=0.949 ; p<.05$ in the "No sound of ice cubes" condition) whatever the interaction with ice cubes whereas the sound of carbonation induced shorter RTs when interacting with the sight or the sound of ice cubes, $p<.01$ $(b=-51.77, t[8]=4.30$, effect size: $r=0.84)$.

\subsection{The influence of both audiovisual interactions and participants' responses on participants' RTs}

Other linear mixed-effects models were fitted considering the variables linked to the stimuli as well as the participants' responses as independent variables and the participants' RTs as the outcome variable. The final model considered $\left(R^{2}{ }_{C}=0.487\right)$ revealed a significant three-way interaction between the different ice cubes conditions and the participants' responses, $\chi 2(11)=18.85, p<.0001$. When the participants categorized the stimuli as fresh, a Tukey post hoc analysis revealed that both the sight $(b=-102.72, t[10]=-6.72$, effect size: $r=0.91)$, and the sound of ice cubes $(b=-72.9, t(12)=-4.52$, effect size: $r=0.79$ ) significantly decreased the participants' RTs, $p<.001$. Moreover, this effect was additive when the two cues were presented simultaneously.

When the participants categorized the stimuli as not fresh, a Tukey post hoc analysis revealed that their RTs were significantly longer in the "Sight of ice cubes" or both Sight and sound of ice cubes conditions ( $p$.001), as compared to the "No ice cubes" or "Sound of ice cubes" only conditions.

Another three-way interaction between the different carbonation conditions and the participants' responses was revealed by the model, $\chi 2[13]=7.04, p=0.03$. A Tukey post hoc analysis revealed that the sight of carbonation always induced significantly longer RTs, $p<.001$ ( $b=66.9, t[6]=6.4$, effect size: $r=0.93$ ), regardless of whether the participants categorized the stimuli as fresh or not fresh. However, the sound of carbonation induced significantly shorter RTs when the participants categorized the stimuli as fresh, $p<.001(b=-58.2, t$ $(13)=-4.12$, effect size: $r=0.75)$. Due to these opposite effects of the sight and sound of carbonation on RTs, no additive effect of carbonation on the participants' RTs was obtained.



FIGURE 3 Mean RTs (in ms) for the three-way interactions between (a) the sight of ice cubes and the different carbonation conditions; (b) the sound of ice cubes and the different carbonation conditions. The error bars represent the SEM. (a) For both solid and hatched black lines, respectively, all means are significantly different from each other. Except in the no carbonation condition, all means in the presence of ice cubes are always significantly different from the means in the absence of ice cubes. (b) For both solid and hatched lines, all means are significantly different from each other. Except in the no carbonation condition, all means in the presence of ice cubes are always significantly different from the means in the absence of ice cubes 


\subsection{The influence of audiovisual interactions on participants' responses}

Generalized linear mixed-effects models were fitted to the data as binary logistic regressions with the participants' responses as the outcome variable, participants as random variable and the congruency variable as nested. When considering the variables linked to the stimuli as independent variables, the final model considered (Tjur's coefficient of discrimination: $D=0.39$, see Tjur, 2009) revealed a significant three-way interaction effect between the different carbonation conditions and the sight of ice cubes on the participants' responses, $\chi 2$ (9) $=252.42, \quad p<.0001$. Another three-way interaction effect between the different ice cubes conditions and the sight of carbonation was revealed by the model, $\chi 2(13)=10.2, p=.0014$. The color of the stimuli did not significantly influence the categorization of freshness $(F=1.26)$. These results are presented in Figure 4 .

The interaction between the different carbonation conditions and the sight of ice cubes appeared to have a significant influence on participants' responses: a Tukey post hoc analysis revealed that in the "No sight of ice cubes" condition, both the sound ( $b=0.66, z=9.6$ ) and sight of carbonation $(b=0.54, z=7.6)$ had a positive effect for
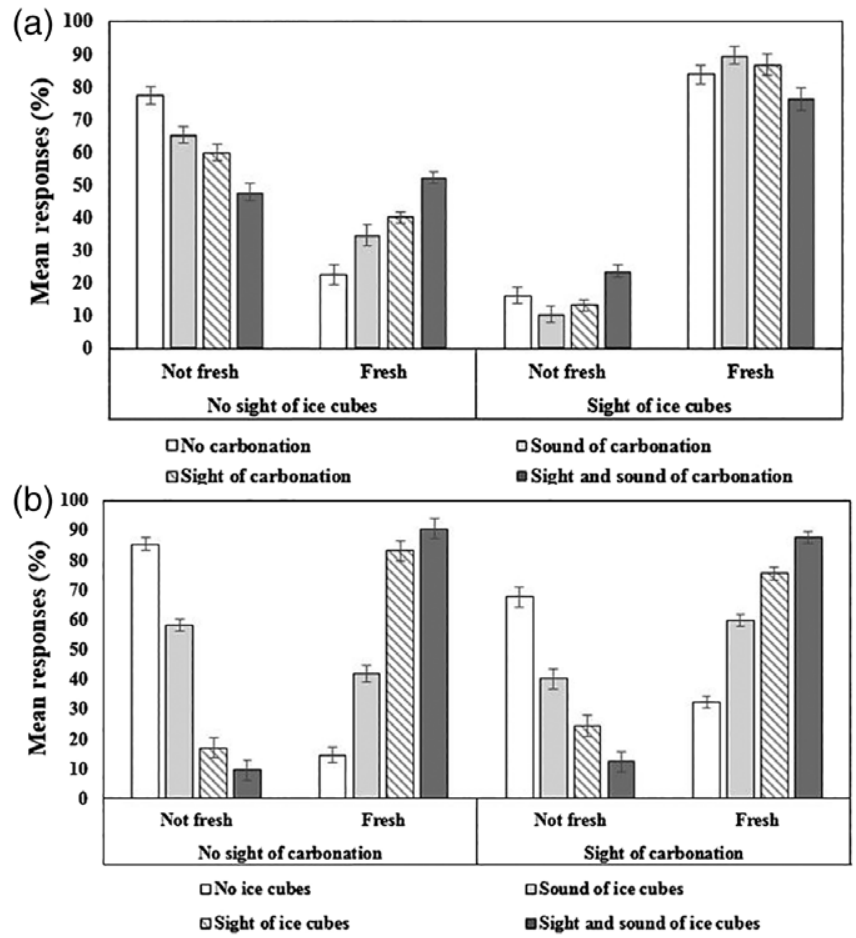

FIGURE 4 Mean responses (\%) for the categorization of freshness according to (a) the different carbonation conditions and the sight of ice cubes; (b) the different ice cubes conditions and the sight of carbonation. $\mathrm{N}=84$ for each condition. The error bars represent the SEM. (a) All the represented means are significantly different from each other, except the comparisons between the light-gray and the hatched bars in the no sight of ice cubes condition and the white and the dark-gray bars in the sight of ice cubes condition, respectively, for not fresh and fresh categories that failed to reach statistical significance. (b) All the represented means are significantly different from each other, except the comparisons between the dark-gray bars in the "No sight of carbonation" condition and those in the "Sight of carbonation" condition, respectively, for not fresh and fresh categories that failed to reach statistical significance categorizing the stimuli as fresh, with an additive effect when the two were presented together, increasing the percentage of responses when the participants categorized the stimuli as fresh (vice versa for not fresh), $p<.001$. However, the percentage of stimuli categorized as fresh did not exceed chance level, whatever the carbonation condition. The sight of ice cubes in the drink induced a strong inclination for participants to categorize the stimuli as fresh $(b=3.27, z=40.9$, $p<.001$ ), whatever the interaction with carbonation. No additive effect of the sight and sound of carbonation was observed in this case since the combination of the sight of ice cubes and the sight of carbonation decreased the proportions of stimuli that were categorized as fresh. The condition in which the beverage was slightly more likely to be categorized as fresh was the one with the sound of carbonation and the sight of ice cubes ( $M=89.64 \%$ ).

Another significant influence on the participants' responses was revealed for the interaction between the different ice cubes conditions and the sound of carbonation: a Tukey post hoc analysis revealed that in the "No sight of carbonation" condition, both the sound $(b=1.43, z=19.8, p<.001)$ and sight of ice cubes $(b=3.27$, $z=40.9, p<.001$ ) had a positive effect for categorizing the stimuli as fresh, with an additive effect when the two were presented together, increasing the percentage of responses when the participants categorized the stimuli as fresh (vice versa for not fresh). In the "Sight of carbonation" condition, the same additive effect of the sight and sound of ice cubes was observed in similar proportions. However, as opposed to the sight of ice cubes, the sight of carbonation did not strongly favor participants' categorization of the stimuli as fresh. As long as ice cubes were visible in the drink, the participants were more likely to categorize the stimuli as fresh in the "No sight of carbonation" condition $(p<.001)$, as opposed to the conditions including the sight of carbonation.

The final model considered revealed another significant threeway interaction between the different carbonation conditions and the sound of ice cubes, $\chi 2(12)=38.72, p<.0001$. In those conditions that included the sound of ice cubes, a similar inclination for participants to categorize the stimuli as fresh more often, whatever the interaction with carbonation, was observed, except that it appeared as less salient as compared to the one induced by the sight of ice cubes, as shown in Figure 4a.

\section{4 | DISCUSSION}

\section{1 | The decrease in participants' RTs depends on both the type and number of perceptual features available}

Interaction effects that can result from the joint presentation of various perceptual features belonging to different sensory modalities have often been studied using speeded classification tasks (see Marks, 2004; Spence, 2018 for reviews). People generally respond more rapidly to multisensory stimuli than to unisensory stimuli. This effect has been characterized as a "statistical facilitation" assuming that the processing time of multisensory stimuli is lower than that of the component unisensory stimuli (Diederich \& Colonius, 2004). Moreover, 
previous studies have highlighted that congruent bimodal stimuli lead to shorter RTs and better accuracy in their responses than incongruent bimodal stimuli (see Spence, 2011). Based on this theoretical and empirical framework, the present study was designed to investigate the influence of congruent and incongruent audiovisual stimuli on the categorization of freshness in beverages. We hypothesized that the perceptual features of interest (i.e., ice cubes, carbonation, and color) would influence the expected freshness of the drinks perceived in mouth.

Overall, the participants' RTs were significantly shorter for congruent bimodal stimuli than for incongruent pairs of auditory and visual stimuli. This result is in line with those obtained in previous studies which have investigated the impact of audiovisual interactions or other types of bimodal or trimodal interactions on participants' RTs (e.g., Evans \& Treisman, 2011; Gallace \& Spence, 2006; Hecht \& Reiner, 2009; Misselhorn, Daume, Engel, \& Friese, 2016). However, our results highlight that this effect depends on both the type and number of perceptual features involved in the audiovisual interaction, as well as on the type of response required from the participants. First, congruent audiovisual interactions linked to temperature (i.e., ice cubes presented visually and auditorily) induced shorter RTs when the participants categorized the stimuli as fresh and this effect was additive. The decrease in the participants' RTs in the presence of congruent bimodal sensory stimuli may be partly explained by the freshness categorization task which may lead to the activation of the content of the freshness category. Hence, the participants may have focused on the different perceptual features they generally associate with freshness. The fact that coldness is likely one of the main sensory contributors to freshness in beverages (as highlighted by our previous online experiment, see footnote 1) can thus partly explain the decrease in the participants' RTs. Further research is needed to disentangle the specificity of this effect toward freshness since congruent stimuli generally facilitate participants' performance independently of the task. The added value of the results obtained here is that, in a freshness categorization task, the sight of ice cubes was found to have a bigger influence than the sound of ice cubes and the sound of carbonation in the decrease of participants' RTs.

On the other hand, the congruency effect inducing shorter RTs for the congruent audiovisual interactions linked to temperature is no longer obtained when there was visible carbonation in the drink, the latter always inducing significantly longer RTs. Thus, in our study, the additive effect of the sound and sight of ice cubes is lost when the stimulus itself becomes more complex. This result suggests that it could be worth going further on research targeting multisensory interactions since the more complex the stimulus becomes (i.e., by adding other sensory cues), the more likely the crossmodal interaction effects can be enhanced or else inhibited. In fact, few studies have considered crossmodal interactions with more than two different perceptual features belonging to the same or different modalities, except those used as distractors in the task. For instance, in Misselhorn et al.'s (2016) study, a bimodal focus was defined and the third modality was considered as a distractor modulating the congruency of the trimodal interaction between auditory, visual, and tactile modalities. The study revealed that the participants' RTs were shorter for congruent trimodal stimuli than for incongruent ones. The fact that different results were obtained in our study can be attributed to the complexity of the stimuli. In fact, the stimuli used sometimes displayed two different perceptual features of interest belonging to the same modality at the same time (e.g., the sight of ice cubes and the sight of carbonation instead of two perceptual features belonging to different modalities). Moreover, the potential influence of confounding variables such as the variation in visual turbidity of the different visual stimuli was not taken into account. It has to be noted that the participants in Misselorn et al.'s study had to respond verbally to the stimuli whereas in our study they answered by pressing one of two response keys. We hypothesize that the modulation of the participants' attention could explain the loss of the additive effect in our study. In fact, the participants' attention could have been directed more to the salient foam on the surface of the liquid than to bubbles present within the liquid itself. In line with this hypothesis, Guinard et al. (1998) found that foam decreased the thirst-quenching character of beers, a psychophysiological factor which has been shown to be positively correlated with the perception of freshness (see Labbe, Almiron-Roig, et al., 2009).

Although the sight of carbonation induced slower RTs whatever the interaction with ice cubes, the sound of carbonation induced shorter RTs in some conditions, in particular when interacting with the sight or the sound of ice cubes. Thus, it would be worth further investigating the influence of the sound of carbonation in freshness categorization tasks, in particular, when interacting with other perceptual features that positively influence people's perception of freshness in beverages.

\subsection{The categorization of freshness is mainly influenced by the sight of ice cubes in a drink}

Congruent bimodal interactions resulted in the participants categorizing the stimuli as fresh, mainly when ice cubes could be seen in the drink whereas minor contributions of the sound of ice cubes and both the sight and sound of carbonation were observed. In fact, as long as ice cubes could be seen in the drink, the participants were more likely to categorize the stimuli as fresh regardless of the carbonation condition. Thus, it would appear that the French participants who took part in the present study gave more weight to perceptual features linked to coldness (i.e., ice cubes) when they were asked to categorize various drinks as fresh or not fresh.

Regarding the influence of carbonation on the way in which the participants categorized the stimuli as fresh, contrasting results were obtained when compared to the results obtained for the participants' RTs for which the sight of carbonation slowed RTs. In fact, the results on the categorization of freshness highlight that the sight of carbonation still positively contributes to the proportion of stimuli that were categorized as fresh. However, this contribution remains minor as compared to the contribution of the sight and sound of ice cubes. It is possible that the small differences in the exact volume of the foam as well as the location of the ice cubes in the glass in each condition may have influenced the allocation of the participants' attention, which could have been more directed to either the ice cubes, the foam, or both according to the condition. Moreover, since the visual stimuli used in our study were not dynamic, we can 
wonder whether the participants considered the bimodal stimuli as referring to one particular drink instead of just two distinct sensory inputs. The present study still lays the groundwork for more ecologically approaches that could take into account various sources of ecological validity: for instance, the use of dynamic stimuli instead of static stimuli (see Gvili et al., 2015). Manipulating other perceptual cues for which participants could infer temperature (e.g., condensation on the glass) and carbonation (e.g., sound of opening of carbonated beverages) would also allow researchers to extend the results of the present study.

\subsection{The influence of the color of the liquid on participants' RTs and the categorization of freshness}

Beyond the influence of audiovisual perceptual features linked to temperature and carbonation, the color of the stimuli was also manipulated. The results failed to show any influence of the color of the stimuli either on the participants' RTs, or on the categorization of freshness. This is not in line, to some extent, with the literature regarding freshness since the results obtained in several studies have highlighted, at least for American consumers, that particular colors of the liquid (clear, orange, and red) positively influenced the perceived freshness of beverages (Clydesdale et al., 1992; Zellner \& Durlach, 2002). However, no consensus emerged concerning the interpretation of brown drinks in these studies. It would seem that the effect of color on the perception of freshness depends on several factors such as the type of measure (quantitative versus qualitative), the corresponding flavor (expected or actually tasted), and likely on the participants' cultural background or, at least, their learned freshness-colors associations (see Wan, Woods, Seoul, Butcher, \& Spence, 2015). One could for instance, imagine that a dark brown liquid might remind people of coffee, whereas the same drink with carbonation would immediately connote a cola drink instead. Finally, similarly to Fenko et al.'s (2009) results, the present findings suggest that the interactions between the different perceptual features used in our study may have caused sensory dominance inducing a minor contribution of color on the categorization of freshness as compared to other sensory cues (see also Hecht \& Reiner, 2009 on sensory dominance).

It is important to note that the present study has certain limitations that should be taken into account in future studies. In relation with the color of the liquid and the potential association with a particular temperature of the beverage (e.g., coffee versus cola drink), it can be noted that some psychological traits associated to well-being might also influenced the participants' performances in our study. For instance, Zhong and Leonardelli (2008) have investigated the relation between the feeling of social exclusion in humans and the desire for warm food and drink. They highlighted that social exclusion is associated with feeling cool and leads to an increased preference for warm liquids (e.g., coffee) as compared to cold liquids (e.g., "icy coke"). Whether such a psychological trait (i.e., social exclusion) may influence participants' performances in a speeded categorization task remains to be investigated.

Furthermore, in order to avoid any association with a particular beverage that the participants could consider as fresh per se (due to branding, labelling, or previously learned associations), no specific information on the beverages that the participants had to categorize (as fresh or not) was provided to our participants. However, it is likely that this lack of information might have induced different kinds of associations leading to inter-individual variability that could perhaps explain the lack of effect of color in our study. In this regards, including the color variable in the design as a between-participants factor might also reduce the possibility to reveal a color effect on freshness judgments. In fact, the number of participants per color was quite small $(N=12)$ and the mechanisms underlying some crossmodal interactions have been shown to be relative and not absolute (see Spence, 2011, on the relative aspect of crossmodal correspondences). Consequently, having color as a between-participants factor did not allow visual comparisons of the different colored beverages in our study.

Moreover, it should be noted that only one saturation of each of the 7 colors was used and that the selection of different saturations might have produced different results. Hence, it remains an open empirical question for future research to determine whether similar results could be obtained toward freshness in more ecological conditions. For instance, the external validity of the findings reported here could be investigated within the context of an advertisement by accommodating both auditory and visual inputs. It would enable to understand how the audiovisual interaction effects that may trigger consumers' freshness expectations translate into the marketplace and whether or not higher levels of perceived freshness increase purchase intention.

Finally, by avoiding the presence of organic ingredients (e.g., mint leaves) and directing the participants' attention to the expected in-mouth freshness that they might perceive from the different beverages, we aimed at constraining the interpretation of the word freshness reducing the association with the aging of organic ingredients or time delay. However, when the participants answered to a beverage that contained visual ice cubes (or visual carbonation) and subsequently answered to visual stimuli with the same color but for which visual ice cubes (or bubbles) were absent, it is possible that they interpreted this sequence as ice cubes' melting (or bubbles vanishing). In this case, the meaning of freshness could have been associated to the time delay between the preparation and the consumption of the drink. Extending this hypothesis, the relation between an increasing time delay and a decreasing perception of freshness of the beverages could also be associated to an increasing perception of the microbial population. It has been shown that this potential source of poisoning may generate incidental disgust in consumers that will reduce their preference for the products they perceive as contaminated (see Hobbs \& Roberts, 1987; Motoki \& Sugiura, 2018).

On the basis of our results, it would appear that an interesting perspective for future investigation would be to address the crossmodal interaction effects that can occur in the categorization of freshness between different kinds of audiovisual perceptual features linked to temperature, carbonation, and color. We believe that the effects resulting from the interaction of visual and auditory cues could be triggered to facilitate consumers' categorization of a given product as fresh. This idea is in line with some empirical studies that have highlighted that audiovisual inputs can significantly influence the final percept of the product experienced (Piqueras-Fiszman \& Spence, 2015; Spence, 2015). However, the influence of other major sensory contributors (e.g., olfactory cues) cannot be disregarded (Martin et al., 2005, see Auvray \& Spence, 2008; Spence, Auvray, \& Smith, 2014, for reviews). Thus, one promising perspective would be to enrich the 
stimulus context by adding sensory contributors beyond the audiovisual in order to generate additional results on the multisensory mechanisms that underlie freshness perception in beverages. Enriching that way the stimulus context by adding, step-by-step, distinct sensory contributors, is required to generalize our results to more ecological contexts. Another interesting follow-up in real consumption context would be to investigate the influence of ambient temperature during the consumers' freshness evaluation of beverages while modifying the products' temperature and color (see Motoki, Saito, Nouchi, Kawashima, \& Sugiura, 2018 who have shown that ambient warm temperature decreases the preference for savory foods). Modulating the ambient temperature might also enable researchers to understand the mechanisms that lead some populations living in warm countries to consider hot beverages (e.g., hot mint tea in North Africa) as fresh or refreshing (see Roque et al., 2018 for the distinction between these two concepts). In addition, it might be interesting in future research to investigate if the color of the liquid plays a bigger role in modulating freshness perception for hot as compared to cold beverages.

\section{5 | CONCLUSIONS}

To summarize, the results of the present study highlight how both visual and auditory perceptual features linked to temperature and carbonation can influence the categorization of freshness in beverages. In particular, a twofold effect has been revealed regarding the congruency of the interaction between the visual and the auditory features. First, the decrease in RTs for congruent bimodal stimuli depends on both the type and number of perceptual features involved as well as on the type of response. In particular, the sight of ice cubes mostly contributed to the decrease in the participants' RTs when the stimuli were categorized as fresh, followed by the sound of ice cubes, and the sound of carbonation. On the other hand, the sight of carbonation induced longer RTs potentially caused by the modulation of the participants' attention. Second, congruent bimodal stimuli that displayed the perceptual features of interest have induced an inclination for participants to be more likely to categorize the stimuli as fresh. In particular, the sight and sound of ice cubes mostly contributed, followed by the sound and sight of carbonation. Even though no effect of color was observed here, we believe that the influence of color on freshness perception certainly depends on several factors such as the type of measure (quantitative versus qualitative), the corresponding flavor (expected or actually tasted), the meaning of freshness that is targeted, and likely on the participants' cultural background or, at least, on their learned freshness-colors associations as well. Thus, further investigations regarding multisensory interactions in the case of freshness perception in beverages could extend the results obtained here to shed new light on the congruency effect and the respective contributions of the perceptual features involved. In particular, if specific mechanisms such as crossmodal correspondences can be triggered upstream (i.e., in anticipation) of the consumption phase, it could positively influence freshness perception and may ultimately increase beverage attractiveness and appreciation.

\section{ACKNOWLEDGMENTS}

We are grateful to Dr. John Prescott for fruitful discussions concerning the experiment, and Dr. Herb Meiselman for his advices on the manuscript. The authors would also like to thank Claire Garrel who was at the origin of this project, M. Philippe Courcoux for his advice concerning the statistical analysis of the data, as well as the reviewers of this manuscript for providing relevant comments of improvement. The project was funded by Pernod Ricard, France, and the French ANRT (Association Nationale de la Recherche et de la Technologie, contract number 2015/0894). The authors have no conflict of interest to declare.

\section{ORCID}

Jérémy Roque (iD) http://orcid.org/0000-0002-8293-4995

Charles Spence (iD) http://orcid.org/0000-0003-2111-072X

\section{REFERENCES}

Auvray, M., \& Spence, C. (2008). The multisensory perception of flavour. Consciousness and Cognition, 17, 1016-1031.

Carvalho, F. R., Moors, P., Wagemans, J., \& Spence, C. (2017). The influence of color on the consumer's experience of beer. Frontiers in Psychology, 8, 2205.

Clydesdale, F. M., Gover, R., Philipsen, D. H., \& Fugardi, C. (1992). The effect of colour on thirst quenching, sweetness, acceptability and flavour intensity in fruit punch flavoured beverages. Journal of Food Quality, 15, 19-38.

Demattè, M. L., Sanabria, D., \& Spence, C. (2007). Olfactory-tactile compatibility effects demonstrated using a variation of the implicit association test. Acta Psychologica, 124, 332-343.

Deroy, O., Fasiello, I., Hayward, V., \& Auvray, M. (2016). Differentiated audio-tactile correspondences in sighted and blind individuals. Journal of Experimental Psychology: Human Perception and Performance, 42, 1204-1214.

Diederich, A., \& Colonius, H. (2004). Bimodal and trimodal multisensory enhancement: Effects of stimulus onset and intensity on reaction time. Perception \& Psychophysics, 66, 1388-1404.

Eccles, R., Du-Plessis, L., Dommels, Y., \& Wilkinson, J. E. (2013). Cold pleasure. Why we like ice drinks, ice-lollies and ice cream. Appetite, 71, 357-360.

Evans, K. K., \& Treisman, A. (2011). Natural cross-modal mappings between visual and auditory features. Journal of Vision, 10, 1-12.

Fenko, A., Schifferstein, H. N. J., Huang, T.-C., \& Hekkert, P. (2009). What makes products fresh: The smell or the colour? Food Quality and Preference, 20, 372-379.

Field, A., Miles, J. N. V., \& Field, Z. (2012). Discovering statistics using R. London: Sage.

Gallace, A., \& Spence, C. (2006). Multisensory synesthetic interactions in the speeded classification of visual size. Perception \& Psychophysics, 68, 1191-1203.

Guinard, J. X., Souchard, A., Picot, M., Rogeaux, M., \& Sieffermann, J. M. (1998). Sensory determinants of the thirst-quenching character of beer. Appetite, 31, 101-115.

Gvili, Y., Tal, A., Amar, M., Hallak, Y., Wansik, B., Giblin, M., \& Bommelaer, C. (2015). Fresh from the tree: Implied motion improves food evaluation. Food Quality and Preference, 46, 160-165.

Hecht, D., \& Reiner, M. (2009). Sensory dominance in combinations of audio, visual and haptic stimuli. Experimental Brain Research, 193, 307-314.

Hobbs, B. C., \& Roberts, D. (1987). Food poisoning and food hygiene (5th ed.). London: Edward Arnold.

Knöferle, K. M., \& Spence, C. (2012). Crossmodal correspondences between sounds and tastes. Psychonomic Bulletin \& Review, 19, 992-1006.

Labbe, D., Almiron-Roig, E., Hudry, J., Leathwood, P., Schifferstein, H. N. J., \& Martin, N. (2009). Sensory basis of refreshing perception: Role of psychophysiological factors and food experience. Physiology \& Behavior, 98, 1-9. 
Labbe, D., Gilbert, F., Antille, N., \& Martin, N. (2009). Sensory determinants of refreshing. Food Quality and Preference, 20, 100-109.

Labbe, D., Martin, N., Le Coutre, J., \& Hudry, J. (2011). Impact of refreshing perception on mood, cognitive performance and brain oscillations: An exploratory study. Food Quality and Preference, 22, 92-100.

Liang, P., Roy, S., Chen, M.-L., \& Zhang, G.-H. (2013). Visual influence of shapes and semantic familiarity on human sweet sensitivity. Behavioural Brain Research, 253, 42-47.

Marks, L. E. (2004). Cross-modal interactions in speeded classification. In G. A. Calvert, C. Spence, \& B. E. Stein (Eds.), Handbook of multisensory processes (pp. 85-105). Cambridge, MA: MIT Press.

Martin, N., Gartenmann, K., Cartier, R., Vaccher, C., Callier, P., Engelen, L., \& Belin, E. (2005). Olfactory cues modulate sensory expectations and actual perceptions of texture and complex sensory attributes. In Abstract book of the sixth Pangborn sensory symposium (09). Oxford, UK: Elsevier.

McEwan, J. A., \& Colwill, J. S. (1996). The sensory assessment of the thirst-quenching characteristics of drinks. Food Quality and Preference, 7, 101-111.

Misselhorn, J., Daume, J., Engel, A. K., \& Friese, U. (2016). A matter of attention: Crossmodal congruence enhances and impairs performance in a novel trimodal matching paradigm. Neuropsychologia, 88, 113-122.

Motoki, K., Saito, T., Nouchi, R., Kawashima, R., \& Sugiura, M. (2018). The paradox of warmth: Ambient warm temperature decreases preference for savory foods. Food Quality and Preference, 69, 1-9.

Motoki, K., \& Sugiura, M. (2018). Disgust, sadness, and appraisal: Disgusted consumers dislike food more than sad ones. Frontiers in Psychology, 9, 76.

Nakagawa, S., \& Schielzeth, H. (2013). A general and simple method for obtaining $\mathrm{R}^{2}$ from generalized linear mixed-effects models. Methods in Ecology and Evolution, 4, 133-142.

Parise, C. V., \& Spence, C. (2012). Audiovisual crossmodal correspondences and sound symbolism: a study using the implicit association test. Experimental Brain Research, 220, 319-333.

Piqueras-Fiszman, B., \& Spence, C. (2015). Sensory expectations based on product-extrinsic food cues: An interdisciplinary review of the empirical evidence and theoretical accounts. Food Quality and Preference, 40, 165-179.

Roque, J., Auvray, M., \& Lafraire, J. (2018). Understanding freshness perception from the cognitive mechanisms of flavor: The case of beverages. Frontiers in Psychology, 8, 2360.

Saint-Eve, A., Déléris, I., Feron, G., Ibarra, D., Guichard, E., \& Souchon, I. (2010). How trigeminal, taste and aroma perceptions are affected in mint-flavored carbonated beverages. Food Quality and Preference, 21, 1026-1033.

Spence, C. (2011). Crossmodal correspondences: A tutorial review. Attention, Perception, \& Psychophysics, 73, 971-995.

Spence, C. (2015). Eating with our ears: Assessing the importance of the sounds of consumption on our perception and enjoyment of multisensory flavour experiences. Flavour, 4, 3.

Spence, C. (2018). Multisensory perception. In J. Wixted (Ed.-in-Chief), J. Serences, The Stevens' handbook of experimental psychology and cognitive neuroscience, 4 th ed. RoutledgeLondon.
Spence, C., Auvray, M., \& Smith, B. (2014). Confusing tastes with flavours. In S. Biggs, M. Matthen, \& D. Stokes (Eds.), Perception and its modalities (pp. 247-276). Oxford, UK: Oxford University Press.

Spence, C., \& Wang, (. Q.). J. (2017). Assessing the impact of closure type on wine ratings and mood. Beverages, 3, 52 .

Tjur, T. (2009). Coefficients of determination in logistic regression models - A new proposal: The coefficient of discrimination. The American Statistician, 63, 366-372.

Velasco, C., Jones, R., King, S., \& Spence, C. (2013). The sound of temperature: What information do pouring sounds convey concerning the temperature of a beverage. Journal of Sensory Studies, 28, 335-345.

Wan, X., Woods, A. T., Seoul, K.-H., Butcher, N., \& Spence, C. (2015). When the shape of the glass influences the flavour associated with a coloured beverage: Evidence from consumers in three countries. Food Quality and Preference, 39, 109-116.

Wang, (. Q.). J., \& Spence, C. (2017). The role of pitch and tempo in sound-temperature crossmodal correspondences. Multisensory Research, 30, 307-320.

Westerink, J., \& Kozlov, S. (2004). Freshness in oral care: Attributes and time-dependency of a multidimensional dynamic concept. Journal of Sensory Studies, 19, 171-192.

Zampini, M., \& Spence, C. (2005). Modifying the multisensory perception of a carbonated beverage using auditory cues. Food Quality and Preference, 16, 632-641.

Zellner, D. A., \& Durlach, P. (2002). What is refreshing? An investigation of the color and other sensory attributes of refreshing foods and beverages. Appetite, 39, 185-186.

Zellner, D. A., \& Durlach, P. (2003). Effect of color on expected and experienced refreshment, intensity, and liking of beverages. The American Journal of Psychology, 116, 633-647.

Zhang, T., Lusk, K., Mirosa, M., \& Oey, I. (2016). Understanding young immigrant Chinese consumers' freshness perceptions of orange juices: A study based on concept evaluation. Food Quality and Preference, 48, 156-165.

Zhong, C. B., \& Leonardelli, G. J. (2008). Cold and lonely: Does social exclusion literally feel cold? Psychological Science, 19, 838-842.

\section{SUPPORTING INFORMATION}

Additional supporting information may be found online in the Supporting Information section at the end of the article. 